\title{
Thoracic spinal osteomyelitis due to Salmonella enteritidis in an immunocompetent mimicking tuberculosis
}

Sir,

In India, tuberculosis is the most common cause of spinal infections. The routinely followed practice in cases of infective spondylitis without instability is to start empirical antitubercular therapy where the clinical picture, radiological, and magnetic resonance imaging (MRI) findings resemble the tuberculosis infection of the spine, although tissue biopsy and culture are done in some cases to confirm the diagnosis. Here, we report a case of spinal infection in a healthy middle-aged immunocompetent man, in whom the clinical findings and the radiological images were similar to tuberculous infection and were misdiagnosed as tuberculous spondylitis on admission and later on, the biopsy and culture of the decompressed specimen surprisingly turned out to be Salmonella osteomyelitis.

A 47-year-old male presented to our outpatient department with mid back pain for about 1 month, which started worsening for the past 4 days before admission. On clinical examination, he was afebrile, normotensive, and there was tenderness over the spinal process of D6 to D9 without any obvious swelling or neurological deficit. The laboratory tests revealed C-reactive protein (CRP) of $9.6 \mathrm{mg} / \mathrm{L}$ and erythrocyte sedimentation rate (ESR) was $50 \mathrm{~mm} / \mathrm{h}$. MRI study showed D7-D8 Spondylodiscitis associated with small pre- and para-vertebral, anterior epidural space abscess [Figures 1 and 2].
An empirical diagnosis of tuberculosis spine (Dorsal spondylitis) was made, and he was started on antituberculous treatment with isoniazid, rifampicin, pyrazinamide, and ethambutol. Computed tomography-guided biopsy done at initial evaluation was inconclusive and showed scanty nonspecific inflammatory cells only. Subsequently, after a few days, as he had severe pain not responding to conservative measures, he was subjected to left posterolateral thoracotomy, D7 D8 corpectomy, and D6-D9 fusion with titanium cage packed with cancellous bone graft, rods, and screws [Figure 3]. Intercostal drainage tube was maintained for 3 days and was removed after ensuring good lung expansion. Postoperatively, his pain was relieved, and he made uneventful recovery.

To our surprise, Histopathological studies revealed infection along with neutrophil infiltration and granulation tissue hyperplasia of the dorsal vertebra with no evidence of tuberculosis. Microbiological reports confirmed Salmonella enteritidis infection in the surgical specimen. Hence, he was diagnosed to have dorsal spondylitis caused by S. enteritidis. Blood culture was negative. Postoperatively, tests for hemoglobinopathy were done and found to be negative. Serum agglutinins were normal.

Based on the antibiotic susceptibility pattern, he was started with levofloxacin and amikacin administered 
through intravenous route for 10 days, followed by oral levofloxacin and co-trimoxazole for 2 weeks. At 1 month

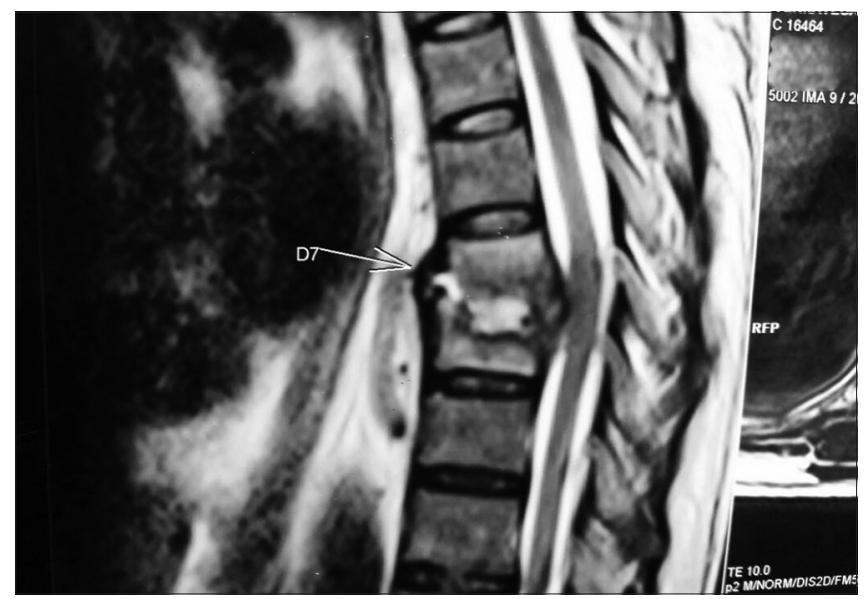

Figure 1: T2-weighted sagittal section of magnetic resonance imaging dorsal spine showing D7-D8 spondylodiscitis with prevertebral and anterior epidural granulation tissue

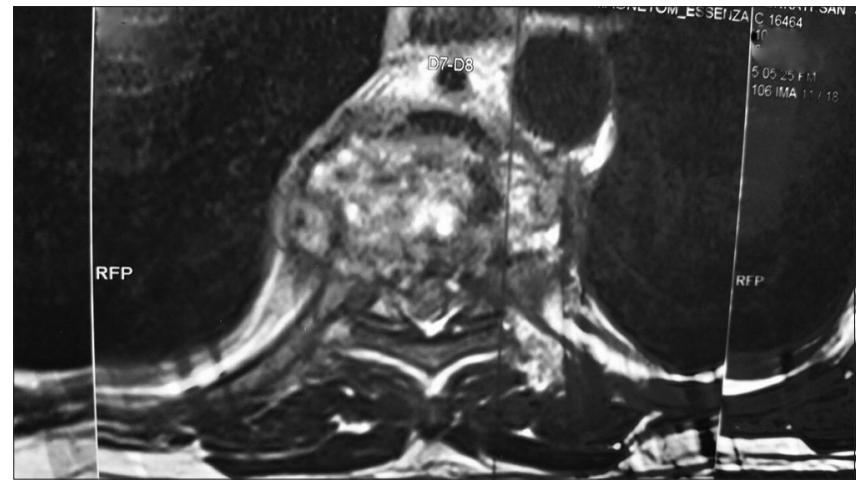

Figure 2: T2-weighted axial section of the magnetic resonance imaging dorsal spine showing destructive spondylitic lesion with pre- and paravertebral granulation tissue

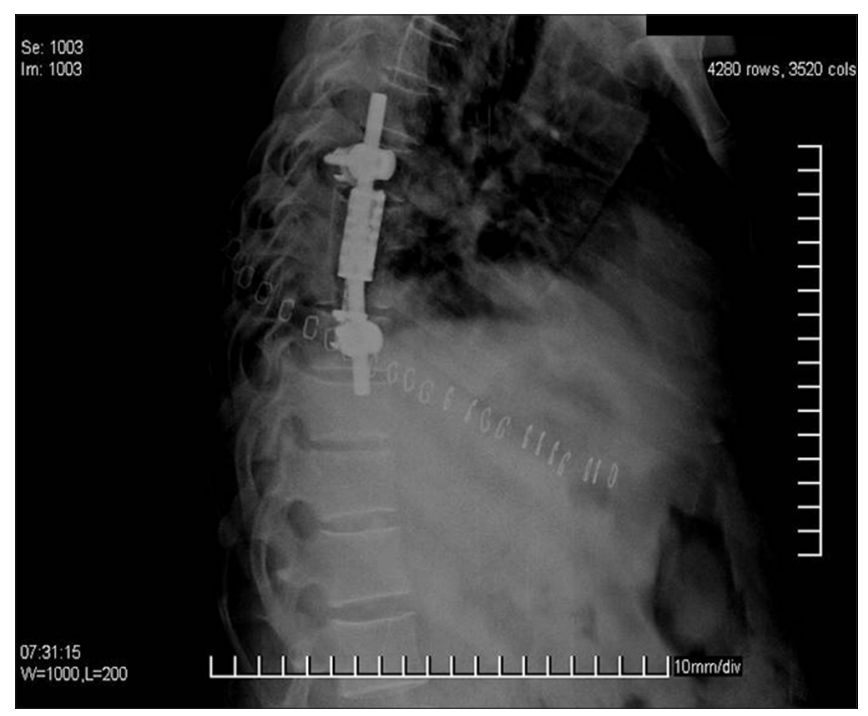

Figure 3: Postoperative X-ray dorsal spine lateral view showing D6D9 fusion follow-up, his back pain was relieved, and his ESR and CRP were within normal range. $\mathrm{X}$-ray done at 3 months follow-up showed fusion from D6 to D9 with implants in situ.

Salmonella species can cause a wide range of human infections, from typhoid fever, gastrointestinal infections to septicemia or may remain as asymptomatic carrier. Pyogenic spondylodiscitis due to Salmonella species is very rare. The spread of infection to the bone may occur because of the hematogenous spread of the bacteria during the unrecognized phases of bacteremia. Salmonella spondylodiscitis is usually common in immunocompromised patients. Salmonella osteomyelitis has been reported in patients with sickle cell disease where sickling occurring in the intestine may lead to the passage of the Salmonella in the gut flora into the blood stream and finally reaching the bone causing infection. ${ }^{[1]}$

However, Salmonella is unusual in patients who have neither sickle cell disease nor who are immunocompromised. Salmonella species causing lumbar spondylodiscitis ${ }^{[2]}$ and cervical spondylitis ${ }^{[3]}$ has been reported in the literature. However, in our case, Salmonella infection of dorsal spine had occurred in an immunocompetent male without any prodromal gastrointestinal symptoms. Laboratory tests revealed no immunological abnormalities. S. enteritidis infection of spine can mimic Guillain - Barre Syndrome, neoplasia, atypical borreliosis, and tuberculosis. ${ }^{[3]} S$. enteritidis often causes fever, diarrhea, and abdominal cramps in the affected individuals and remote cases can have some immunodepression factors. Salmonella infections usually respond well to the antibiotic treatment. The commonly used antibiotics are ciprofloxacin, chloramphenicol, ceftriaxone, ampicillin, etc. The duration of treatment varies from 6 weeks to 9 weeks. Multidrug resistance has been observed in few cases.

On the other hand, the clinical and radiological presentation of the case mimicked tuberculous spondylitis. Kim et al., in their study analyzed and compared the clinical, radiological, and laboratory features of both pyogenic spondylitis and tuberculous spondylitis and reported that previous invasive spinal procedures, preceding bacteremia, fever, elevated white blood cells count, CRP, ESR, etc., are all the indications of pyogenic spondylitis. ${ }^{[4]}$

While there are a few reports of Salmonella spondylodiscitis caused by Salmonella typhi and paratyphi ${ }^{[2,5]}$ S. enteritidis causing spontaneous spondylodiscitis has been only rarely reported. ${ }^{[3]}$ This organism normally inhabits the 
gastrointestinal system but what factors were responsible for the invasion and destruction of the vertebrae needs further evaluation.

It is very difficult to differentiate Salmonella and tuberculous spondylitis as both have similar clinicoradiological and epidemiological findings. The exact diagnosis largely depends on the recovery of the organism by culture methods. MRI findings may not clearly differentiate tuberculous spondylitis and pyogenic spondylitis. Well-defined abnormal signal in the paraspinal space, presence of paraspinal or intraspinal abscess, the wall of the abscess being thin and smooth, and the involvement of thoracic spines are the features indicative of tuberculous spondylitis.

In this case also, the abscess wall was thin and smooth, dorsal spines were involved with well-defined abnormal paraspinal signals which lead to the misdiagnosis of the case to be tuberculous spondylitis [Figures 1 and 2] before the surgical intervention. Furthermore, as tuberculosis of spine is very common in our country, a provisional diagnosis of tuberculosis was made, and empirical antitubercular therapy was initiated and continued until the biopsy report proved it to be Salmonella infection. Our experience with this patient illustrates the need for tissue biopsy and confirmation of the diagnosis. The prevalent practice of empirical antituberculous treatment for spinal infections in regions endemic for tuberculosis ${ }^{[6]}$ cannot be considered scientific. On the other hand, such kind of mismanagement might be harmful in the interest of the patients by endangering them to the risk of neurological deterioration.

The treating clinician needs to have a high degree of suspicion to diagnose Salmonella vertebral osteomyelitis. The diagnosis largely depends on the isolation of the organism by appropriate culture methods. Even in regions endemic for tuberculosis, an alternative diagnosis should be entertained. Tissue biopsy and culture must be obtained in all cases of spinal infections.

\section{Financial support and sponsorship}

Nil.

\section{Conflicts of interest}

There are no conflicts of interest.

K. Lakshmi, R. Santhanam ${ }^{1}$

Departments of Microbiology and ${ }^{1}$ Neurosurgery, Sree Balaji Medical College and Hospital, Bharath University, Chennai, Tamil Nadu, India

Address for correspondence: Dr. R. Santhanam,

Department of Neurosurgery, Sree Balaji Medical College and Hospital, Bharath University, Chennai, Tamil Nadu, India. E-mail: drsanthanam79@yahoo.co.in

\section{References}

1. Givner LB, Luddy RE, Schwartz AD. Etiology of osteomyelitis in patients with major sickle hemoglobinopathies. J Pediatr 1981;99:411-3.

2. Kumar P, Mahmoodi SM, Kalaparambil Moosa N, Edgar M, Samt HA, Hussain RA, et al. Salmonella paratyphi spondylitis: A case report. Eur Spine J 2008;17:754-5.

3. Feng ZY, Guo F, Chen Z. Literature review and clinical presentation of cervical spondylitis due to Salmonella enteritidis in immunocompetent. Asian Spine J 2014;8:206-10.

4. Kim CJ, Song KH, Jeon JH, Park WB, Park SW, Kim HB, et al. A comparative study of pyogenic and tuberculous spondylodiscitis. Spine (Phila Pa 1976) 2010;35:E1096-100.

5. Amritanand R, Venkatesh K, Sundararaj GD. Salmonella spondylodiscitis in the immunocompetent: Our experience with eleven patients. Spine (Phila Pa 1976) 2010;35:E1317-21.

6. Yasaratne B, Wijesinghe S, Madegedara R. Spinal tuberculosis: A study of the disease pattern, diagnosis and outcome of medical management in Sri Lanka. Indian J Tuberc 2013;60:208-16.

This is an open access article distributed under the terms of the Creative Commons Attribution-NonCommercial-ShareAlike 3.0 License, which allows others to remix, tweak, and build upon the work non-commercially, as long as the author is credited and the new creations are licensed under the identical terms.

\begin{tabular}{|l|l|}
\hline \multicolumn{2}{|c|}{ Access this article online } \\
\hline Quick Response Code: & Website: \\
\hline & www.ruralneuropractice.com \\
\cline { 2 - 2 } & \\
\hline
\end{tabular}

How to cite this article: Lakshmi K, Santhanam R. Thoracic spinal osteomyelitis due to Salmonella enteritidis in an immunocompetent mimicking tuberculosis. J Neurosci Rural Pract 2016;7:317-9. 